\title{
The contribution of pharmacokinetics and pharmacodynamics to clinical anaesthesia care
}

\author{
Donald R. Stanskj MiD
}

\section{Defining dose requirement, pharmacokinetics and pharmacodynamics}

Clinical anaesthetists administer doses of drugs to obtain various degrees of drug effect. Some anaesthetic drug effects, such as muscle relaxation, are relatively easy to quantitate, whereas others such as anaesthetic depth are much more difficult. Anaesthetic dose requirement can be defined as the dose of drug that produces a defined degree of effect. For example, an $\mathrm{ED}_{50}$ would represent the dose of a drug that results in 50 per cent of the maximal drug effect, whereas an $E_{95}$ is the dose that creates 95 per cent of the maximal drug effect. Alternatively, the dose requirement can be expressed as the dose that results in 50 or 95 per cent of a group of patients reaching a defined drug effect, i.e., no response to a specific stimuli. The dose requirement is determined by two pharmacological processes: pharmacokinetics, which is the relationship between dose and drug plasma concentration and pharmacodynamics, the relationship between drug plasma concentration and degree of dtug effect. Pharmacokinetics characterizes those physiological or phamacological processes that govern the resultant drug plasma concentration or "what the body does to the drug." Pharmacodynamics represents the relationship between drug plasma concentration, the drug concentration at the site of action and the actual occurrence of a measurable drug effect. Pharmacodynamics represents "what the drug does to the body."

\section{Basic Terminology}

Pharmacokinetics and pharmacodynamics are critically dependant on the appropriate measurement technology. Pharmacokinetic research involves measurement of the drug concentrations in biological fluids. Pharmacodynamic research involves measurement of the degree of drug effect. In pharmacokinetic research, one uses mathematical models to describe the interaction of the body's physiological forces with the drug. Five basic pharmacokinetic parameters can be used to describe any drug. ${ }^{1-3}$ These include the initial and steady-state distribution volumes, distributional and metabolic clearances and the terminal elimination half-life.
The distribution volume represents the theoretical body space with which the drug has equilibrated. The initial distribution volume generally reflects blood volume and rapidly perfused (vessel-rich) tissues. The initial distribution volume will govern the peak plasma concentrations after a rapid intravenous bolus. The steady-state distribution volume is a global measure of the overall distribution of drug to all of the body tissues. A large steady-state distribution volume indicates that a significant amount of drug has partitioned into the tissues with generally higher tissue concentrations relative to plasma. The small steadystate distribution volume reflects very limited tissue penetration with drug confined to the blood volume and interstitial fluid. Drug clearance is analogous to the clinical concept of creatinine clearance: the volume of plasma that is completely removed of drug per unit time. Distributional clearance represents a measure of the rate of drug movement from the initial distribution volume to peripheral tissue compartments. Generally, distributional clearance is governed by blood flow to the tissues and is responsible for the initial rapid decline of the drug plasma concentration after an intravenous bolus. The metabolic clearance of the drug is a reflection of the rate of drug metabolism and elimination from the body. The metabolic clearance can involve both the hepatic metabolism to pharmacologically inactive compounds or the excretion of unchanged drug by the kidney. Distributional clearance reflects movement of drug from rapidly equilibrating tissues to more slowly equilibrating tissues within the

Departments of Anesthesia and Medicine (Clinical Pharmacology) Stanford University School of Medicine and Anesthesiology Service, Palo Alto Vetcrans' Administration Medical Center, Palo Alto, California.

Address correspondence to: Dr. Donald R. Stanski, Ancsthesiology Service (112A), Veterans' Administration Medical Center, 3801 Miranda Avenue, Palo Alto, California 94304.

Supported by the National Institute of Aging (RO1-AGO4594, PO1-AGO-3104), the Veterans' Administration Research Grants, and the Anesthesia/Pharmacology Research Foundation. 
body, whereas the metabolic clearance is a measure of the removal of the drug from the body. The terminal elimination half-life reflects the time for one-half of the drug to be removed from the body. The elimination half-life is governed by the metabolic clearance and the steady-state distribution volume. A high metabolic clearance will decrease the terminal elimination half-life while a large steady-state distribution volume will increase the terminal elimination half-life.

Pharmacodynamic descriptors include the maximal degree of drug effect that can occur $\left(E_{\max }\right)$ and the steady-state plasma concentration that results in one-half of the maximal effect $\left(\mathrm{EC}_{50}\right)$. This latter variable can be used as a measure of tissue sensitivity or responsiveness. An additional pharmacodynamic variable involves the half-time of blood:effect site equilibration. This is a measure of how rapidly the drug moves from plasma into the site of action and will be a major factor in determining the onset of drug effect. To obtain the above pharmacokinetic and pharmacodynamic descriptors, it is necessary to gather both plasma concentration and drug effect data from an individual subject. One then uses the appropriate mathematical models with some form of regression data analysis to derive the pharmacokinetic and dynamic descriptors.

\section{How can pharmacokinetics and pharmacodynamics improve clinical care?}

Pharmacokinetic and pharmacodynamic information is the scientific basis for the clinical use of drugs in anaesthetic practice. This knowledge can be used in the following ways.

Understanding the clinical drug effect or dose/response relationship within and between drag groups

There are three important clinical drug measures of the effect: the onset, degree of peak effect, and rate of recovery or dissipation of drug effect. Let us use three narcotic drugs (morphine, fentanyl, alfentanil) as an example of integrating the clinical profiles and dose response relationship with pharmacokinetic and dynamic data. The clinical variables are indicated in Table I; Table II presents the pharmacokinetic variables, while Table III

TABLE I Clinical narcotic profiles for postoperative pain relief

\begin{tabular}{lcll}
\hline & $\begin{array}{l}\text { Equipotent } \\
\text { dosage }\end{array}$ & $\begin{array}{l}\text { Onset of } \\
\text { peak effect } \\
\text { (min) }\end{array}$ & $\begin{array}{l}\text { Recovery to } \\
\text { minimat effect } \\
\text { (hr) }\end{array}$ \\
\hline Morphine & $10 \mathrm{mg}$ & 15 to 30 & 2 to 4 \\
Fentanyl & $10 \mu \mathrm{g}$ & 4 to 8 & 1 to \\
Alfentanil & $750 \mu \mathrm{g}$ & 1 to 2 & 101 \\
\hline
\end{tabular}

TABLE II Representative phamacokinetic profiles

\begin{tabular}{llll}
\hline & $\begin{array}{l}\text { Elimination } \\
\text { half-lfe } \\
(\mathrm{hr})\end{array}$ & $\begin{array}{l}\text { Sieady-state } \\
\text { distribution } \\
\text { volume } \\
\left(\mathrm{L} \cdot \mathrm{kg}^{-1}\right)\end{array}$ & $\begin{array}{l}\text { Metabolic } \\
\text { clearance } \\
\left(\mathrm{ml} \cdot \mathrm{kg}^{-1} \cdot \mathrm{min}^{-}\right)\end{array}$ \\
\hline Morphine & 2 to 3 & 2 to 4 & 10 to 23 \\
Fentanyl & 3 to 6 & 3 to & 8 to 15 \\
Alfentani & 1 to 3 & 0.4 to 0.8 & 3 to 8 \\
\hline
\end{tabular}

TABLE III Physiochemical factors

\begin{tabular}{llcc}
\hline & Morphine & Fentanyl & Alfentanil \\
\hline $\begin{array}{l}\text { \% Unionized at pH 7.4 } \\
\text { Free fraction in human plasma }\end{array}$ & 23 & 8.5 & 89 \\
$\begin{array}{c}\text { Oetanol:Water partition } \\
\text { coefficient at pH 7.4 }\end{array}$ & 1 & $16 \%$ & $8 \%$ \\
$\begin{array}{c}\text { Brain to blood partition } \\
\text { coefficient at steady-state } \\
\text { estimated from animal data }\end{array}$ & not & 960 & 130 \\
\hline
\end{tabular}

indicates relevant physicochemical properties. Alfentanil has the most rapid onset of narcotic effect while morphine is the slowest. ${ }^{4}$ Physicochemical properties can explain this difference in onset of drug effect. Alfentanil has adequate lipid solubility and a high unionized fraction with a relatively low partitioning or solubility in the brain. Alfentanil can rapidly penetrate the blood:brain barrier and equilibrate with narcotic receptors with minimal non-receptor tissue uptake. Morphine, however, has relatively low lipid-solubility and therefore blood:brain barrier penetration and diffusion to the receptor site is rate-limiting. The peak degree of narcotic effect for these opioids is relatively similar at equipotent doses, although the time course is very different to achieve this peak effect. As more opioid is given, a more profound degree of narcotic effect can occur to the point of causing complete anaesthesia (i.e. high-dose opioids for cardiac surgery). The recovery of narcotic effect for these opioids is dependant on the administered dose. At lower doses, redistribution mechanisms from the brain and spinal cord to other tissues that do not contain opioid receptors is an important mechanism to explain the short duration of narcotic effect. This is especially important for the more-lipophilic compounds (fentanyl, alfentanil) that can rapidly penetrate membrane tissues. Morphine has the lowest and slowest degree of redistribution because of its low lipid solubility and slow membrane transfer. Morphine is removed slowly from its site of action. Recovery of drug effect is also very dependant upon the terminal elimination half-life of the drug. At high doses, fentanyl's duration of narcotic effect approximates morphine because redistribution is no longer present and both nar- 
cotics have intermediate length elimination half-lives. Alfentanil has the shortest elimination half-life with the most rapid recovery of narcotic effect. While this example represents a relatively abbreviated overview of complex narcotic clinical pharmacology, it is possible to use pharmacokinetic and dynamic concepts to provide a rational understanding of the clinical profiles of drugs used in anaesthetic practice.

\section{Characterizing the effect of altered physiological states on drug effect}

Pharmacokinetic and pharmacodynamic concepts can be used to characterize how altered physiological or pharmacological states effect the dose requirement, pharmacokinetics, and pharmacodynamics of anaesthetic drugs. For example, with increasing age, the anaesthetic requirement for thiopental, etomidate, fentanyl and alfentanil decreases..$^{5-7}$ Lower doses are needed in the elderly, compared with young patients. By measuring EEG dose requirement, plasma concentrations and EEG drug effect in young and old patients, it has been possible to determine that altered distribution pharmacokinetics in the elderly explains in part the decreased dose requirement of the aged for the two intravenous anaesthetics (thiopentone and etomidate), ${ }^{5.6}$ In contrast, fentanyl and alfentani], demonstrate an enhanced brain sensitivity with increasing age. ${ }^{7}$ Distributional pharmacokinetics are not altered for fentanyl and alfentanil in the elderly. Metabolic clearance does decrease with increasing age for alfentanil, but this does not explain the altered dose requirement.

Many other examples are available whereby disease states alter drug pharmacokinetics. In renal failure, the duration of muscle relaxant effect for pancuronium and d-tubocurarine is markedly prolonged. Because of this marked prolongation of drug effect, much lower doses are required to have an acceptable duration of muscle relaxant effect. Pharmacokinetic studies have shown that both pancuronium and d-tubocurarine have a moderate degree of elimination via the kidneys. ${ }^{8.9}$ Renal failure thus decreases the metabolic clearance and prolongs the terminal elimination half-life. This explains the prolonged duration of drug effect in this clinical situation. In contrast, the two newer relaxants, atracurium and vecuronium, do not have their pharmacokinetics altered with renal disease. ${ }^{10.11}$ Because of this, it is possible to use these relaxants in comparable doses to normal patients and have an cxpected and predictable duration of drug effect.

Disease states and age can also change pharmacodynamic relationships. With increasing age, the MAC (minimum alveolar concentration) of potent inhalational anaesthetics decreases. ${ }^{12}$ This results from increased brain sensitivity to the inhalational anaesthetics. By understanding how disease alters the drug dose requirement, pharmacokinetics and pharmacodynamics, the anaesthetist can more rationally approach an individual patient. By knowing that renal failure markedly prolongs duration of drug effect, one can anticipate this and appropriately change the dosing strategy. In a similar fashion, one can choose drugs whereby the pharmacokinetics and dynamics are not altered by the changed physiological state.

\section{Implementing new approaches to anaesthetic dosing} The two previous subheadings have discussed how pharmacokinetics and pharmacodynamics have improved our understanding of traditional approaches to anaesthetic drug administration. It is possible, however, to use pharmacokinetic and pharmacodynamic coneepts to actually implement anaesthetic drug dosing. Pharmacodynamic research has defined therapeutic plasma concentrations of fentanyl and alfentanil under various clinical situations. The initial loading dose of a drug is going to be governed by the initial and steady-state distribution volumes. The maintenance infusion rate will be given by the metabolic clearance. The ultimate example of using pharmacodynamics to dose drugs during anaesthesia involves the use of computer-driven pumps driven by pharmacokinetic models. ${ }^{13}$ In this situation, the anaesthetist first provides an appropriate pharmacokinetic model and representative kinetic data. After choosing target plasma concentration to be achieved, a computer driven purnp then determines the administration rate at frequent (10- to 15-second) intervals needed to achieve these target concentrations. A computer/pump interface is needed for this new drug delivery concept.

Limitations of pharmacokinetics and pharmacodynamics Pharmacokinetic and pharmacodynamic research is capable of quantitating the relationship between dose, plasma concentration and drug effect for any one individual patient or larger groups of patients. However, this is frequently cone subsequent to the administration of the drug and anaesthetic because analytical measurement of drug plasma concentrations are generally not available intraoperatively. Additionally, there is a small number of anaesthetic drugs where direct measurement of the drug effect is relevant and practical. Research has established that a moderate degree of variability occurs in patients for both pharmacokinetic and pharmacodynamic data. While it is possible to identify factors (i.e., age, weight, sex) that explain some of this variability, it is generally not possible to completely eliminate it. We frequently do not understand why one patient will have a very different degree of response compared to another. This is a clear limitation of 
phamacokinetic and pharmacodynamic research. One ultimate goal would be continuous non-invasive measures of the drug effect that are reliable and predictable, similar to the degree of twitch tension for muscle relaxants. If equivalent measures were ayailable for the narcotics, intravenous and inhalational anaesthetics, then all drug administration could be guided by the degree of drug effect with minimal pharmacokinetic considerations.

In summary, pharmacokinetic and pharmacodynamic research has many broad implications for anaesthetic care. Pharmacokinetic and pharmacodynamic information represents the underlying scientific basis for the administration of anaesthetic drugs to patients. Research allows us to understand how and why drug dosage has to be changed in altered physiologic states. Finally, improved methods of dng administration can be performed using kinetic and dynamic concepts. Pharmacokinetics and dynamics will, however, never replace the important human components and judgement involved in anaesthetic practice. Because we do not have absolute foolproof measures of anaesthetic drug effect that can be continuously monitored, the anaesthestist's training and judgement will always be critical in providing optimal, safe patient care.

\section{References}

1 Stanski DR, Watkins WD. Drug Disposition in Anesthesia. New York: Grune \& Stratton, 1982.

2 Wood $M$, Wood AJJ (Eds). Drugs and Anesthesia: Pharmacology for Anesthesiologists. Baltimore: Willians and Wilkins, 1982.

3 Prys-Roberts C, Hug CC Jr (Eds). Pharmacokinetics of Anaesthetic Practice. London, Blackwell Scientific Publications, 1984.

4 Scott JC, Ponganis KV, Stanski DR. EEG yuantitation of narcotic effect: a comparison of fentanyl and aifentanil. Anesthesiology 1985, 62: 234-41.

5 Homer TD and Stanski $D R$. The effect of increased age on thiopental disposition and anesthetic requirement. Anesthesiology 1985; 62: 714-24.

6 Arden JR, Holley FO, Stanski DR. Increased sensitivity to etomidate in the elderly: initial distribution $v s$ altered brain sensitivity. Anesthesiology 1986; 65: 19-27.

7 Scott JC and Stanski DR. Decreased fentanyl and alfentanil dose requirement with age: a simultaneous pharmacokinetic and pharmacodynamic evaluation. I Pharmacol Exp Ther 1987; 240: 159-66.

8 Miller RD. Matteo RS. Benet LZ, Sohn YJ. The pharmacokinetics of $d$-tubocurarine in the man with and without renal failure. J Pharmacol Exp Ther 1977; 202: 1-7.
9 Somozyi A, Shanks CA, Triggs EJ. The effects of renal failuse on the disposition and neuromuscular blocking action of pancuronium bromide. Eur J Clin Pharmacol 1977; 12 23-9.

10 Fahey MR, Morris RB, Miller RD, Nguyen TL, Upton RA. Pharmacokinetics of ORG NC 45 (Norcuron) in patients with and without renal failure. Br J Anaesth 1981; 53: 1049-53.

11 Htnter $J M$, Jones $R S$, Utting $J E$. Use of atracurium in patients with no renal function. Br J Araesth 1982: 54: 1251-8.

12 Stevens WC, Dolan WM, Gibbon.s RT, Eger EI. Minimum alveolar concentration (MAC) of isoflurane with and without nitrous oxide in patients of various ages. Anesthesiology 1975; 42: 197-200.

13 Stanski DR. The clinical pharmacology of alfentanil. Eur J Anaesth 1987; Supp 1: 3-12 